\title{
First Use of High Charge States for Mass Measurements of Short-lived Nuclides in a Penning Trap
}

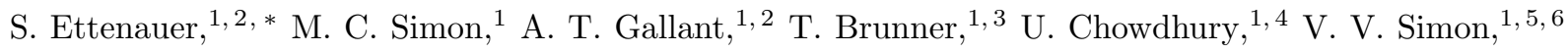 \\ M. Brodeur,,${ }^{1,2,7}$ A. Chaudhuri, ${ }^{1}$ E. Mané, ${ }^{1}$ C. Andreoiu,${ }^{8}$ G. Audi, ${ }^{9}$ J. R. Crespo López-Urrutia, ${ }^{5}$ P. Delheij,${ }^{1}$ \\ G. Gwinner, ${ }^{4}$ A. Lapierre, ${ }^{1,7}$ D. Lunney, ${ }^{1,9}$ M. R. Pearson, ${ }^{1}$ R. Ringle,${ }^{7}$ J. Ullrich,${ }^{5}$ and J. Dilling ${ }^{1,2}$ \\ ${ }^{1}$ TRIUMF, 4004 Wesbrook Mall, Vancouver, BC V6T 2A3, Canada \\ ${ }^{2}$ Department of Physics and Astronomy, University of British Columbia, Vancouver, BC V6T 1Z1, Canada \\ ${ }^{3}$ Physik Department E12, Technische Universität München, D-85748 Garching, Germany \\ ${ }^{4}$ Department of Physics and Astronomy, University of Manitoba, Winnipeg, MB R3T 2N2, Canada \\ ${ }^{5}$ Max-Planck-Institut für Kernphysik, Saupfercheckweg 1, 69117 Heidelberg, Germany \\ ${ }^{6}$ Ruprecht-Karls-Universität, Heidelberg, Germany \\ ${ }^{7}$ National Superconducting Cyclotron Laboratory, Michigan State University, East Lansing, MI 48824, USA \\ ${ }^{8}$ Department of Chemistry, Simon Fraser University, Burnaby, BC V5A 1S6, Canada \\ ${ }^{9}$ CSNSM-IN2P3-CNRS, Université Paris 11, 91405 Orsay, France
}

(Dated: November 14, 2018)

\begin{abstract}
Penning trap mass measurements of short-lived nuclides have been performed for the first time with highly-charged ions (HCI), using the TITAN facility at TRIUMF. Compared to singly-charged ions, this provides an improvement in experimental precision that scales with the charge state $q$. Neutron-deficient Rb-isotopes have been charge bred in an electron beam ion trap to $q=8-12+$ prior to injection into the Penning trap. In combination with the Ramsey excitation scheme, this unique setup creating low energy, highly-charged ions at a radioactive beam facility opens the door to unrivalled precision with gains of 1-2 orders of magnitude. The method is particularly suited for short-lived nuclides such as the superallowed $\beta$ emitter ${ }^{74} \mathrm{Rb}\left(T_{1 / 2}=65 \mathrm{~ms}\right)$. The determination of its atomic mass and an improved $Q_{E C}$-value are presented.

PACS numbers: 21.10.Dr,24.80.+y, 27.50.+e
\end{abstract}

Since their introduction into rare isotope research over twenty years ago [1, 2], Penning traps have made major contributions to the exploration of the nuclear mass surface as evidenced by the large number of existing and proposed facilities 3 ] as well as the wealth of experimental results [4. Advances in experimental techniques now allow measurements for virtually all low energy, rare isotope beams as Penning traps have been able to access nuclides with half-lives below $10 \mathrm{~ms}$ [5] as well as superheavies with production yields of less than 1 particle per second [6. The widespread success of Penning traps is due to their precision following

$$
\frac{\delta m}{m} \propto \frac{m}{q B T_{\text {rf }} \sqrt{N_{\text {ion }}}}
$$

[7, where $\delta m / m$ is the achievable relative precision in mass $m, q$ is the ion's charge state and $B$ is the magnetic field strength. The measurement time $T_{\text {rf }}$ and the number of ions $N_{\text {ion }}$ are limited by a nuclide's half-life and possibly by its production yield at radioactive beam facilities and efficiency of the spectrometer. Measurements are generally performed with singly-charged ions (SCI) or in special cases, where coupled to a gas stopper cell, with $q=2+$. Penning trap mass studies utilizing highlycharged ions (HCI) have been successfully pioneered with stable nuclides [8]. Here the requirements of high efficiency and short measurement times are less relevant compared to working with radioactive ions. In the realm of rare isotope science with Penning traps, HCI repre- sent a thus far unexplored opportunity to improve the experimental precision further circumventing constraints imposed by short half-lives and lower yields when probing the limits of nuclear existence.

The superallowed $\beta$ emitter ${ }^{74} \mathrm{Rb}$ is a prime example where a short half-life of only $65 \mathrm{~ms}$ poses a real challenge to experiment. Despite several Penning trap mass measurements 9 11, the total transition energy, $Q_{E C}$, still contributes significantly to the uncertainty of its corrected $\mathcal{F} t$-value, only surpassed by theoretical uncertainties of the isospin-symmetry breaking corrections $\delta_{C}[12$. These have recently been reduced by experimentally providing the ${ }^{74} \mathrm{Rb}$ rms charge radius as an input for the calculation of $\delta_{C}\left[13\right.$. The $Q_{E C}$-value and $\delta_{c}$ are now close to sharing the same weight to the total uncertainty of the $\mathcal{F} t$-value. Among all superallowed $\beta$ emitters used to extract $V_{\mathrm{ud}}$ of the Cabibbo-Kobayashi-Maskawa (CKM) matrix [12] ${ }^{74} \mathrm{Rb}$ has the highest atomic number, $Z$. It is hence of special importance in attempts to distinguish between conflicting nuclear models since $\delta_{C}$ approximately scales as $Z^{2}$ [14, 15]. In this letter we present the first Penning trap mass measurements of short-lived HCI performed, with TRIUMF's Ion Trap for Atomic and Nuclear science (TITAN) [16], including a successful mass determination of ${ }^{74} \mathrm{Rb}^{8+}$.

Neutron-deficient $\mathrm{Rb}$ isotopes were produced at TRIUMF's ISAC facility 17 by bombarding a Nb-target with a $98 \mu \mathrm{A}, 500 \mathrm{MeV}$ proton beam from the cyclotron. The surface-ionized Rb beam was accelerated to $20 \mathrm{keV}$ 


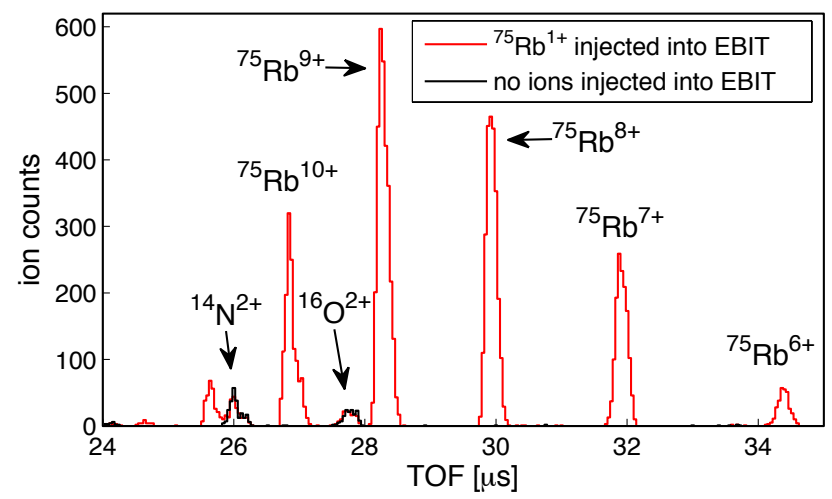

FIG. 1: (color online) TOF spectrum of 500 ion bunches of radioactive ${ }^{75} \mathrm{Rb}$ extracted from the EBIT with an $800 \mathrm{~ns}$ extraction pulse after $35 \mathrm{~ms}$ of charge breeding with a $10 \mathrm{~mA}$, $2.5 \mathrm{keV}$ electron beam. ${ }^{16} \mathrm{O}^{2+}$ and ${ }^{14} \mathrm{~N}^{2+}$ are due to ionized and further charge-bred residual gas in the EBIT.

and mass separated prior to injection into TITAN's radiofrequency quadrupole (RFQ) cooler and buncher 18. where the ions were accumulated and cooled through collision with a He buffer gas. Extracted ion bunches were transferred with a beam energy of about $2 \mathrm{keV}$ into the electron beam ion trap (EBIT) 19. Operated with a 10 $\mathrm{mA}, 2.5 \mathrm{keV}$ electron beam, the EBIT confined the ions radially by the space-charge of the electron beam and an axial magnetic field of $3 \mathrm{~T}$. The central, trapping drift tube was biased (at $U_{\text {trap }}$ ) slightly below the beam energy to remove most of the ions' kinetic energy. To provide confinement in the axial direction, a bias voltage of $\approx 100$ $\mathrm{V}$ above the central drift tube was applied to the neighbouring drift tubes, one of which was lowered during the ions' capture and extraction. Through electron-impact ionization the initially singly charged ions were charge bred to higher charge states. Due to a kinetic energy of $q \cdot U_{\text {trap }}$ after extraction from the EBIT, different charge states $q_{i}$ can be identified via time of flight (TOF) as illustrated in Fig 1. At a fixed electron beam setting, a certain charge state can be maximized in abundance by optimizing the charge breeding time. For instance, the number of $\mathrm{Rb}$ ions with $q=8+$ reached its maximum at a flat plateau of $\approx 20-27$ ms of breeding. A BradburyNielson ion gate (BNG) 20, allowed the selection of one charge state by opening the gate for 300-500 ns during the beam transport from the EBIT to TITAN's measurement Penning trap (MPET). In the MPET, the ion's cyclotron frequency $\nu_{c}=q B /(2 \pi m)$ was determined by the time-of-flight ion-cyclotron resonance (TOF-ICR) technique [21 23. Typical time-of-flight ion-cyclotron resonances recorded during these measurements are shown in Fig 2(a) and (c). At the TOF minimum the rf-frequency $\nu_{\mathrm{rf}}$ equals $\nu_{c}$. The width of a resonance, $\Delta \nu_{\mathrm{fwhm}}$, is solely governed by the duration $T_{\mathrm{rf}}$ of the rf-field [25] but is independent of $q, m$, or $B$. Hence, the gain in relative precision $\delta m / m=\delta \nu_{c} / \nu_{c}$ when utilizing $\mathrm{HCI}$ is due to
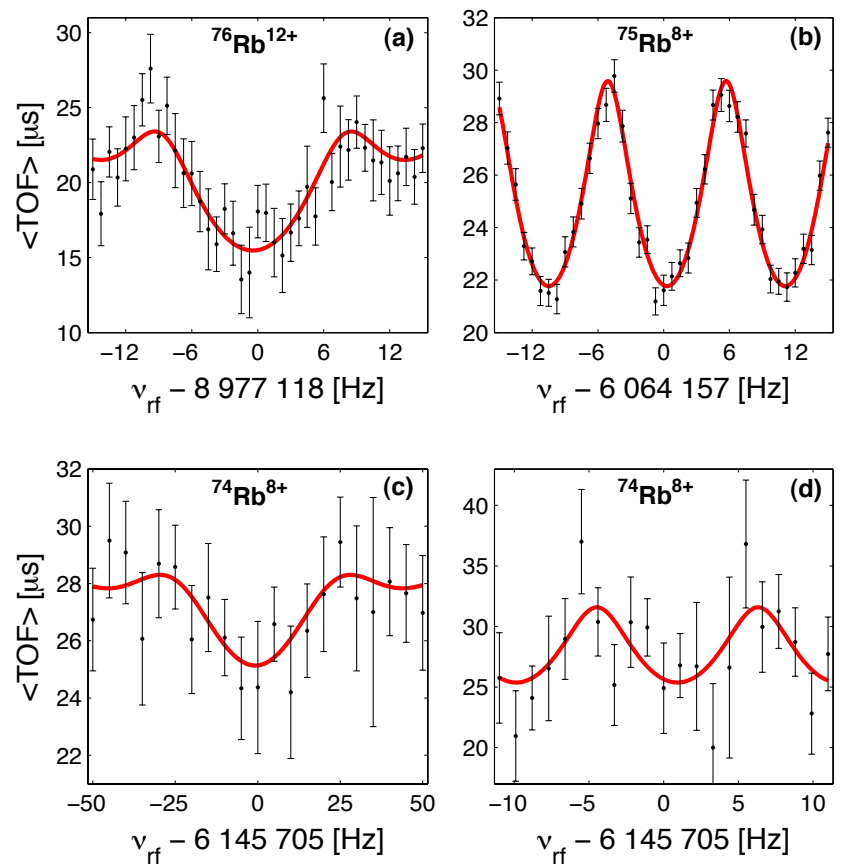

FIG. 2: (color online) Time-of-flight ion-cyclotron resonances for Rb-isotopes in charge state $q=+8$ and $q=+12$. During (a) and (c) the rf-field was continuously applied for $T_{\mathrm{rf}}=97$ $\mathrm{ms}$ and $30 \mathrm{~ms}$, respectively, and a Ramsey excitation scheme with 6-85-6 ms was utilized in (b) and (d). The (red) lines represent the fit to the theoretical line shapes [22, 24].

the larger $\nu_{c}$ for a given $\Delta \nu_{\text {fwhm }}$.

As demonstrated recently, a reduction by a factor of 23 in $\Delta \nu_{\text {fwhm }}$ can be achieved by the Ramsey method of separated oscillatory fields, in which the rf-field is applied during two pulses separated by a waiting period [24, 26. This technique is now used at TITAN, where it can be combined with the advantages offered by HCI. In Fig 2(b) and (d), a Ramsey excitation scheme with two $6 \mathrm{~ms}$ rf-pulses separated by a $85 \mathrm{~ms}$ waiting period has been employed (denoted as 6-85-6 ms throughout the paper). With a charge state $q=9+$ in addition to the Ramsey excitation a gain in precision of $\delta \nu_{c} / \nu_{c}$ by a factor of about 20 has been achieved compared to the conventional technique. A factor of 36 is possible when optimizing our implementation of the Ramsey technique to the performance reported in [27] and using $q=12+$, the highest charge state for which a measurement has been performed.

Due to the $\sqrt{N_{\text {ions }}} \cdot q$ dependence of the achievable precision (see Eq. 1), HCI are favourable as long as the loss in efficiency caused by the charge breeding is smaller than $q^{2}$. However, these measurements must be performed with only a few ions (1-5) at a time. If ${ }^{75,76} \mathrm{Rb}$ had been done with SCI, beam attenuation would have been required. Hence, a lower efficiency for ${ }^{75,76} \mathrm{Rb}^{+8}$ could be compensated by reducing this beam attenuation. The total number of ions is affected by the breeding in three 
TABLE I: Mean frequency ratios $\bar{R}$ of ${ }^{76,75,74} \mathrm{Rb}^{q+}$ and ${ }^{74} \mathrm{Ga}^{8+}$ with respect to ${ }^{85} \mathrm{Rb}^{9+}$. Where applicable, the ratio and error are the result of a count-class analysis. Uncertainties are displayed as (statistical) and $\{$ stat. + systematics $\}$.

\begin{tabular}{cllr}
\hline \hline species & excitation $[\mathrm{ms}]$ & $\bar{R}=\nu_{c}^{\text {ref }} / \nu_{c}^{\text {meas }}$ & \#meas \\
\hline${ }^{76} \mathrm{Rb}^{8+}$ & 97 (conventional) & $1.006067401(15)$ & 5 \\
& 6-85-6 (Ramsey) & $1.006067422(12)$ & 4 \\
& previous combined & $1.006067414\{22\}$ & \\
\hline${ }^{76} \mathrm{Rb}^{12+}$ & 97 (conventional) & $0.670692259(23)$ & 1 \\
\hline${ }^{75} \mathrm{Rb}^{8+}$ & $6-85-6$ (Ramsey) & $0.992864003(10)\{25\}^{a}$ & 5 \\
\hline${ }^{74} \mathrm{Rb}^{8+}$ & 30 (conventional) & $0.979689909(318)^{b}$ & 4 \\
& 6-85-6 (Ramsey) & $0.979689552(79)\{98\}^{b c}$ & 3 \\
& 20(dip)6-65-6(Ram) & $0.97968966(10)\{13\}^{b c}$ & 2 \\
\hline${ }^{74} \mathrm{Ga}^{8+}$ & previous combined & $0.979689609\{86\}$ & \\
\hline \hline
\end{tabular}

${ }^{a} \nu_{c}^{\text {meas }}$ was determined for ${ }^{75} \mathrm{Rb}^{8+}$ by a $40-17-40 \mathrm{~ms}$ Ramsey scheme, in which the sidebands are less pronounced [24].

${ }^{b}$ Too few ions to perform a count-class analysis.

${ }^{c}$ Too few data to study TOF- range dependence. As an upper limit the TOF range dependence for ${ }^{74} \mathrm{Ga}^{8+}$ was added in quadrature.

ways: (1) non-unity efficiency for the chosen charge state due to the charge-state distribution (see Fig 1), (2) increased beam emittance causing a reduced transport and trapping efficiency at MPET, and (3) loss of ions due the radioactive decay in the EBIT. For the ${ }^{74} \mathrm{Rb}$ measurement, ions were kept for $23 \mathrm{~ms}$ in the $\operatorname{EBIT}\left(\sim 0.35 \cdot T_{1 / 2}\right)$ and the breeding was done in parallel to a measurement in MPET.

Since HCI are more likely to exchange charge with the residual gas in MPET, the use of HCI demands severe vacuum requirements compared to SCI. When an ion recombines with one or more electrons during the measurement period, its cyclotron frequency changes and the ion is subsequently unaffected by the rf-excitation scheme. These partially recombined ions add to the detected background reducing the sensitivity and possibly induce frequency shifts, e.g. through ion-ion interactions. In preparation for these measurements, we baked the trap, the vacuum vessel, and the extraction beamline. Despite an improved vacuum of $\approx 6 \cdot 10^{-11}$ mbar in the MPET vacuum section, charge exchange occurred, and in the TOF spectrum we observed an increasing abundance of $\mathrm{H}_{2}^{+}$with longer storage time. Even though the current vacuum allowed the recording of a TOF resonance of ${ }^{76} \mathrm{Rb}^{8+}$ with $T_{\mathrm{rf}}=1 \mathrm{~s}$, ions were typically trapped for $97 \mathrm{~ms}$ in MPET as a compromise between increased precision and recombination for longer $T_{\mathrm{rf}}$. A summary of

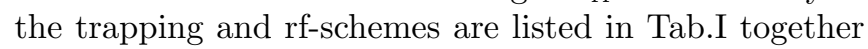
with the measured frequency ratio $R=\nu_{c}^{\text {ref }} / \nu_{c}^{\text {meas }}$ to the reference ion, ${ }^{85} \mathrm{Rb}^{9+} .{ }^{85} \mathrm{Rb}^{+}$was delivered at the same beam energy from TRIUMF's off-line ion source (OLIS) 28. A fraction of the mass $A=74$ beam from ISAC was ${ }^{74} \mathrm{Ga}$, and we have determined its mass as well. In order to purify the beam and to push the contaminant out of the trap, a dipole rf-field at the reduced cyclotron frequency $\nu_{+}$3. of ${ }^{74} \mathrm{Ga}^{8+}$ was applied for $20 \mathrm{~ms}$ during one set of ${ }^{74} \mathrm{Rb}$ measurements.

To avoid potential frequency shifts induced by ion-ion interactions, isobaric contamination, or charge exchange, we only considered ion bunches with 5 or less detected ions per detection cycle and performed a count-class analysis [29] where statistics allowed. For all $A=74$ measurements, the low count rate made it rare that two or more ions were stored at the same time. To be conservative, we have nevertheless added the difference in $\nu_{c}$ between 1 and 1-5 detected ions per ion bunch to the systematic uncertainty. Ions of different charge states were produced due to charge exchange in MPET as mentioned above and extracted onto the MPET detection multichannel plate (MCP), but they could not be resolved in TOF. Thus, the cut on the TOF range was varied in the analysis, and when shifts in $R$ were observed, they were added in quadrature as systematic uncertainties. By far the largest of these shifts was found in ${ }^{74} \mathrm{Ga}$, where it accounts for $60 \mathrm{ppb}$. Other systematic effects due to improper electric field compensation, misalignment between magnetic and trap axes, or harmonic distortions of the electrode structure as well as relativistic effects, were minimized by choosing a reference ion, ${ }^{85} \mathrm{Rb}^{9+}$, with similar $m / q$. Remaining errors were investigated by measuring ${ }^{85} \mathrm{Rb}^{10,8+}$ and ${ }^{87} \mathrm{Rb}^{9+}$ versus ${ }^{85} \mathrm{Rb}^{9+}$ in various experimental settings. Within one standard deviation, all of these measured $R$ agree with the literature values constraining these systematic uncertainties to less than 42 ppb for ${ }^{74} \mathrm{Rb}^{8+}$ and $20 \mathrm{ppb}$ for the other online measurements with $q=8+$. The different upper limits are due to a turbo pump failure that necessiated the reconditioning of the electron beam in the EBIT and subsequent retuning of the injection into MPET. According to 30, 31, the image charges do not alter the measurement of $\nu_{c}$. All systematic uncertainties, including correlations in $R$ due to shared reference frequency measurements, will be discussed in detail in a forthcoming publication.

With the measured $\bar{R}$, the atomic masses of the respective nuclides are calculated in Tab. [I] taking into account the total electron binding energies $(0.5,0.7$, and $1.6 \mathrm{keV}$ for $\mathrm{Rb}^{8,9,12+}[36$, respectively, as well as $0.6 \mathrm{keV}$ for $\left.\mathrm{Ga}^{8+}[37]\right)$. We also performed a complete atomic mass evaluation based on the procedures in [33], but adding the electron binding energies to the linear equations which were neglected in previous evaluations. Our results are in agreement with ISOLTRAP's measurements [11, 32] (see Fig 3). Due to the use of HCI and the Ramsey excitation they are comparable in precision despite our significantly shorter measurement time ( $<20 \mathrm{~h}$ for $\left.{ }^{74} \mathrm{Rb}\right)$. Combined with the ISOLTRAP mass value, the total transition energy, $Q_{E C}$, of the superallowed decay in ${ }^{74} \mathrm{Rb}$ is $10416.8(3.9) \mathrm{keV}$, an improvement by $\approx 12 \%$. This re- 


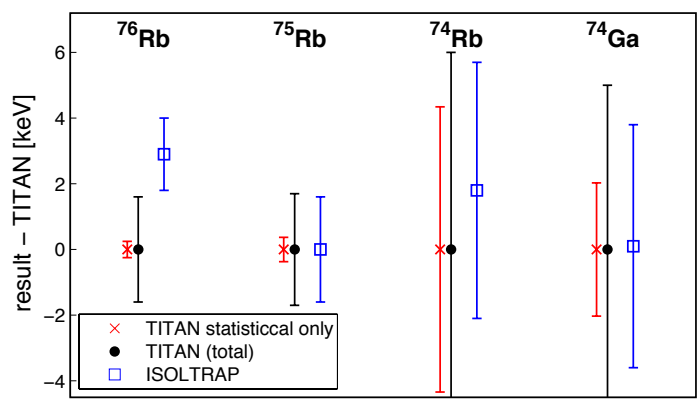

FIG. 3: (color online) Atomic masses of ${ }^{76,75,74} \mathrm{Rb}$ and ${ }^{74} \mathrm{Ga}$ in comparison to their respective ISOLTRAP measurements [11, 32. Statistical uncertainties are based on fits of ion bunches with 1-5 detected ions but without a count-class analysis

TABLE II: Mass excess for measured nuclides. ${ }^{76} \mathrm{Rb}^{12+}$ was a feasibility test of higher charge states. Due to its larger systematic uncertainty, only those with $q=8+$ were considered.

\begin{tabular}{lcc}
\hline \hline nuclide & this work $[\mathrm{keV}]$ & mass evaluation $[\mathrm{keV}]$ \\
\hline${ }^{76} \mathrm{Rb}$ & $-60481.0(1.6)$ & $-60479.1(0.9)$ \\
${ }^{75} \mathrm{Rb}$ & $-57218.7(1.7)$ & $-57218.7(1.2)$ \\
${ }^{74} \mathrm{Rb}$ & $-51916.5(6.0)$ & $-51916.0(3.0)^{a}$ \\
${ }^{74} \mathrm{Ga}$ & $-68049.7(5.0)$ & $-68049.6(3.0)^{b}$ \\
\hline \hline
\end{tabular}

${ }^{a} 33$ included a mass value determined from the $\mathcal{F} t$ value of other superallowed $\beta$ emitters and ${ }^{74} \mathrm{Rb}$ 's half-life and branching ratio [34. This evaluation does the same with an updated Q-value estimate of 10413.8(7.0) keV [35] based on [12. For the discussion of superallowed decays, we do of course not consider this estimate.

${ }^{b}$ Yield measurements determined an isomer to ground state ratio of about 1:190.

sults in a statistical rate function of $f=47283(93)$ [35] and translates together with the recent improvements in $\delta_{C}$ due the laser spectroscopy work [13 to a corrected $\mathcal{F} t$-value of $3077(11) \mathrm{s}$, when considering $\delta_{C}$ based on shell model calculations with Wood-Saxon radial wavefunctions [12]. To reduce its uncertainty further, a new mass measurement of ${ }^{74} \mathrm{Rb}$ and its daughter, ${ }^{74} \mathrm{Kr}$, is planned with charge states up to $q \approx 30+$, reachable by a more intense electron beam $\left(I_{e}=400 \mathrm{~mA}\right)$. However, to take full advantage of HCI, it will be necessary to gain better control over systematic effects. Work is underway to improve further the MPET vacuum to avoid charge exchange and its associated uncertainties. It was demonstrated that $m / q$ dependent shifts at TITAN for SCI are at the level of a few ppb [23, 38, and an experimental confirmation of this accuracy for HCI is expected. In light of the purly statistical uncertainties of ${ }^{76,75} \mathrm{Rb}$ in Fig 3 , such a new measurement could provide knowledge of ${ }^{74} \mathrm{Rb}$ 's Q-value within $0.5 \mathrm{keV}$. Uncertainties of half-life, branching ratio (BR), or theoretical corrections would then surpass the $Q_{E C}$-value's contribution to the $\mathcal{F} t$-value's error, stimulating new BR measurements. At this level of experimental precision $f$ 's uncer- tainty in ${ }^{74} \mathrm{Rb}$ would be dominated by theory and not by the $Q_{E C}$-value [35, 39]. Perhaps most importantly, more stringent comparisons of conflicting theoretical models of $\delta_{c}$ similar to [15] could challenge perceived consistencies between a set of $\delta_{C}$-calculations, experimental results, and the conserved vector current hypothesis since ${ }^{74} \mathrm{Rb}$, with its largest $\delta_{C}$ among all superallowed $\beta$ emitters, would carry particular weight were it not limited by the current precision in its $Q_{E C}$-value.

In summary, Penning trap mass measurements of highlycharged, short-lived nuclides have been performed for the first time opening a new class of on-line mass measurements with potentially up to 2 orders of magnitude improved precision versus conventional SCI-TOF-ICR spectroscopy when combined with the Ramsey excitation. This is essential for fundamental symmetries studies, such as presented here for the superallowed $\beta$ emitter ${ }^{74} \mathrm{Rb}$. For nuclear structure and nuclear astrophysics, where the experimental precision is already sufficient, this novel technique will reduce the measurement time and thus allow one to map the nuclear mass landscape more quickly. In addition, the same precision can be achieved for lower production yields and/or shorter halflives. At this time, TITAN is unique in providing the possibility for high charge states in Penning trap mass spectroscopy of radionuclides.

This work has been supported by the Natural Sciences and Engineering Research Council of Canada and the National Research Council of Canada. We would like to thank the TRIUMF technical staff, especially M. Good. We are very grateful to I.S. Towner for discussions and his calculations. S.E. acknowledges support from the Vanier CGS , T.B. from the Evangelisches Studienwerk e.V. Villigst, and V. V. S. from the Deutsche Studienstiftung.

* Corresponding author: sette@triumf.ca

[1] G. Bollen et al., Hyperfine Interactions 38, 793 (1987).

[2] H. Stolzenberg et al., Phys. Rev. Lett. 65, 3104 (1990).

[3] K. Blaum, Physics Reports 425, 1 (2006).

[4] K. Blaum and M. Block, Hyp. Int. 194, 65 (2009).

[5] M. Smith et al., Phys. Rev. Lett. 101, 202501 (2008).

[6] M. Block et al., Nature 463, 785 (2010).

[7] G. Bollen, Nuclear Physics A 693, 3 (2001).

[8] C. Carlberg et al., Phys. Rev. Lett. 83, 4506 (1999).

[9] F. Herfurth et al., EPJ A 15, 17 (2002).

[10] A. Kellerbauer et al., Phys. Rev. Lett. 93, 072502 (2004).

[11] A. Kellerbauer et al., Phys. Rev. C 76, 045504 (2007).

[12] J. C. Hardy and I. S. Towner, PRC 79, 055502 (2009).

[13] E. Mané et al., submitted to Phys. Rev. Lett. (2011).

[14] G. F. Grinyer et al., Nucl. Instr. Meth. A 622, 236 (2010).

[15] I. S. Towner and J. C. Hardy, PRC 82, 065501 (2010).

[16] J. Dilling et al., IJMS 251, 198 (2006).

[17] M. Dombsky et al., Rev. Sci. Instrum. 71, 978 (2000).

[18] T. Brunner et al., arXiv:1107.2187 (2011).

[19] A. Lapierre et al., Nucl. Instr. Meth. A 624, 54 (2010). 
[20] T. Brunner et al., arXiv:1107.4010, accepted for publication in IJMS (2011).

[21] G. Gräff et al., Zeitschrift für Physik A 297, 35 (1980).

[22] M. König et al., IJMS 142, 95 (1995).

[23] M. Brodeur et al., Physical Review C 80, 044318 (2009).

[24] M. Kretzschmar, IJMS 264, 122 (2007).

[25] G. Bollen et al., J. App.Phys. 68, 4355 (1990).

[26] S. George et al., Phys. Rev. Lett. 98, 162501 (2007).

[27] S. George et al., IJMS 264, 110 (2007).

[28] K. Jayamanna et al., Rev. Sci. Instr. 79, 02C711 (2008).

[29] A. Kellerbauer et al., Eur. Phys. J. D 22, 53 (2003).
[30] R. S. Van Dyck et al., Phys. Rev. A 40, 6308 (1989).

[31] I. Bergström et al., NIM A 487, 618 (2002).

[32] C. Guénaut et al., Phys. Rev. C 75, 044303 (2007).

[33] G. Audi et al., Nuclear Physics A 729, 337 (2003).

[34] A. Piechaczek et al., Phys. Rev. C 67, 051305(R) (2003).

[35] I. S. Towner, private communications (2011).

[36] J. E. Sansonetti, J.Phys.Chem.Ref.Data 35, 301 (2006).

[37] T. Shirai et al., J.Phys.Chem.Ref. Data 36, 509 (2007).

[38] M. Brodeur et al., submitted to to IJMS (2011).

[39] J. C. Hardy and I. S. Towner, PRC 71, 055501 (2005). 